\title{
Ancient DNA from lakes: Insights into plant response to past climate changes
}

\author{
Laura Parducci
}

\begin{abstract}
Considerable advances during the last decade in DNA sequencing technology and bioinformatics have enabled in-depth study of the genome from a larger number of ancient samples derived from a larger variety of substrates and environments. In particular, extraction and analysis of ancient DNA from lake sediments has provided important clues regarding the evolution of past environments in relation to climate change. This information is essential for understanding the importance of climate and plants' dispersal ability in determining their distributional ranges, and for predicting the response of plants to ongoing climate change.
\end{abstract}

\begin{abstract}
Ancient lakes
Ancient lake sediments store biological, chemical, and physical information that allow for a detailed reconstruction of past environmental and climatic changes. Biological micro- and macrofossil remains, combined with adequate chronologies, provide not only information on changes in patterns, structure, and diversity of past ecosystems, but can also be used to reconstruct past temperature and precipitation. Despite a wealth of information on past environmental and climatic changes that has been obtained from lake sedimentary records during the past decades, paleoecologists are aware that fossil assemblages represent only a tiny fraction of all the organisms that existed at a certain time in the past (due to e.g. postmortem processes, differential decomposition, or depositional changes). In addition, each paleoecological method has its specific limitations (e.g. diatoms may partly be dissolved; high pollen producers obscure the abundance of other plant species; some species are better preserved than others in sediments).
\end{abstract}

\section{Large-scale sequencing data from lakes Recent advances in ancient DNA (aDNA) analyses now make use of large-scale sequencing technologies, which parallelize the sequencing process and produce mil- lions of sequencing reads simultaneously. In this way, DNA sequenced from soils offer an unprecedented opportunity to unravel a complementary and larger spectrum of plant taxa compared to those identified by micro- or macrofossil analyses alone (Taberlet et al. 2018). They also allow identification of plants at lower taxonomic levels than conventional paleoecological proxies (Willerslev et al. 2014; Pedersen et al. 2016; Parducci et al. 2019). However, DNA from soil mainly rep- resents taxa growing a few meters from the sampling point (Edwards et al. 2018).}

Lakes, on the other hand, have large catchment areas and recent studies have suggested that plant aDNA from lacustrine sediments is a more powerful tool for paleo floristic reconstruction (Parducci et al. 2017; Giguet-Covex et al. 2019). In particular, sediment records from small lakes are excellent archives for molecular studies, as the effects of disturbances are low and seem to represent the surrounding terrestrial environment well (Fig. 1). As such, aDNA studies of lake sediments have the potential to revise and complement our understanding of how plant species and communities responded in the past to long- and short-term environmental changes. At the species level, this allows us to assess the effect of climate change over hundreds of generations, while at the community level it permits the investigation of the structure of ancient communities (dominant versus rare species) and their correlation with temperature, precipitation, and biotic interactions. This information is essential for understanding the importance of climate and dispersal ability of plants in determining past ranges and for predicting the response of ranges to ongoing and future climate changes.

\section{Plants' responses to climate}

There are several examples of questions that can be addressed by using aDNA analyses from lakes to tackle issues related to plants' responses to climate and that can help us understand what we can do in the face of future changes. One in particular is the question concerning the Reid's paradox of rapid migration of plants (Reid et al. 1899). This is the observation that the speed of forest recolonization process after the last Quaternary glaciation (from ca. 12 cal. kyr

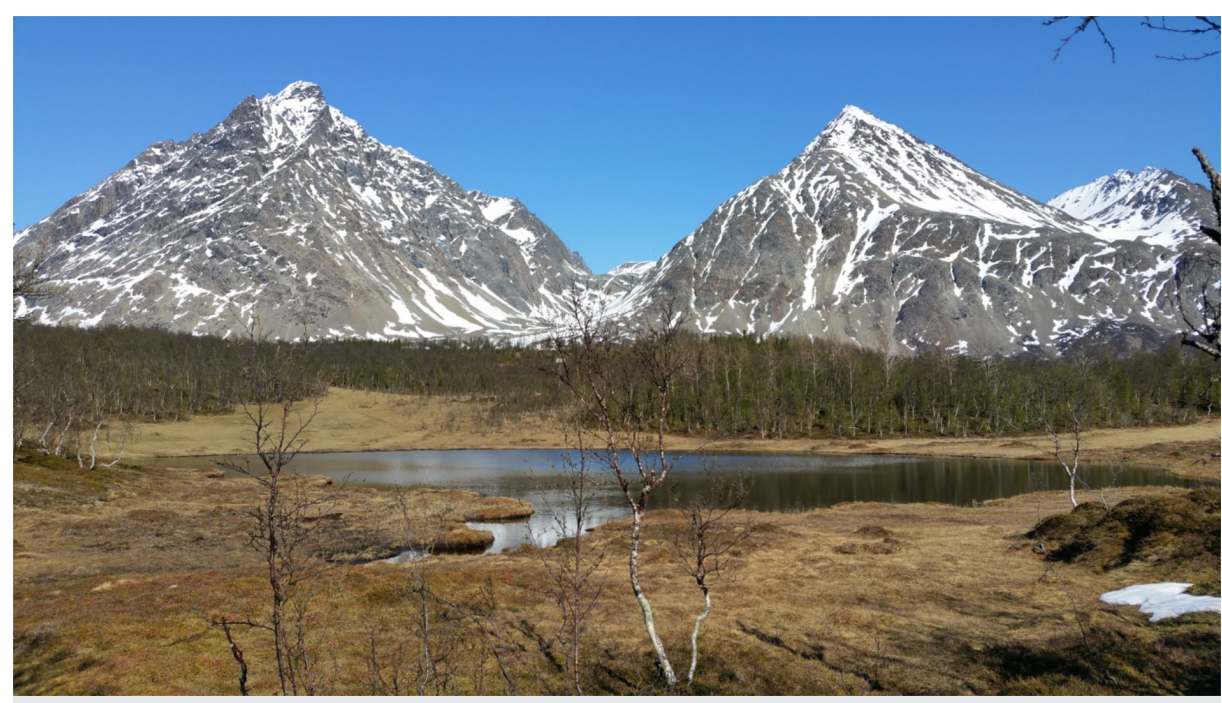

Figure 1: Rundvatnet, Lyngen, Tromsø, Norway (Photo credit: Inger Greve Alsos).
$\mathrm{BP})$ is faster (115-550 m/yr; Feurdean et al. 2013) than it would be expected, based on life histories and ability to transport seeds and fruits of the species involved (Clark et al. 1998). Fast recolonization can be explained by rare long-distance dispersal events mediated by birds and wind and promise well for future populations suggesting that plants can do so again in response to global warming, but alternative explanations need to be investigated thoroughly.

One alternative explanation, though controversial (Tzedakis et al. 2013), is that cold-tolerant tree species survived at high latitudes in small isolated microrefugia and recolonized locally once the ice retreated (Stewart et al. 2010). Recently, several lines of evidence based on fossil analysis, species distribution modeling, and phylogeographical surveys have been used to propose the existence of these small microrefugia at northern latitudes in the Northern Scandinavia, in central Sweden, several megafossils of mountain birch (Betula pubescens Ehrh. ssp. tortuosa (Ledeb.) Nyman), Norway spruce (Picea abies (L.) Karst.), and Scots pine (Pinus sylvestris (L.)) have been recovered earlier than expected $(14,11$, and 11.7 kyr BP, respectively; Kullman 2002), and in the same region today, hundreds of clonal Hemisphere (e.g. Napier et al. 2019). In 


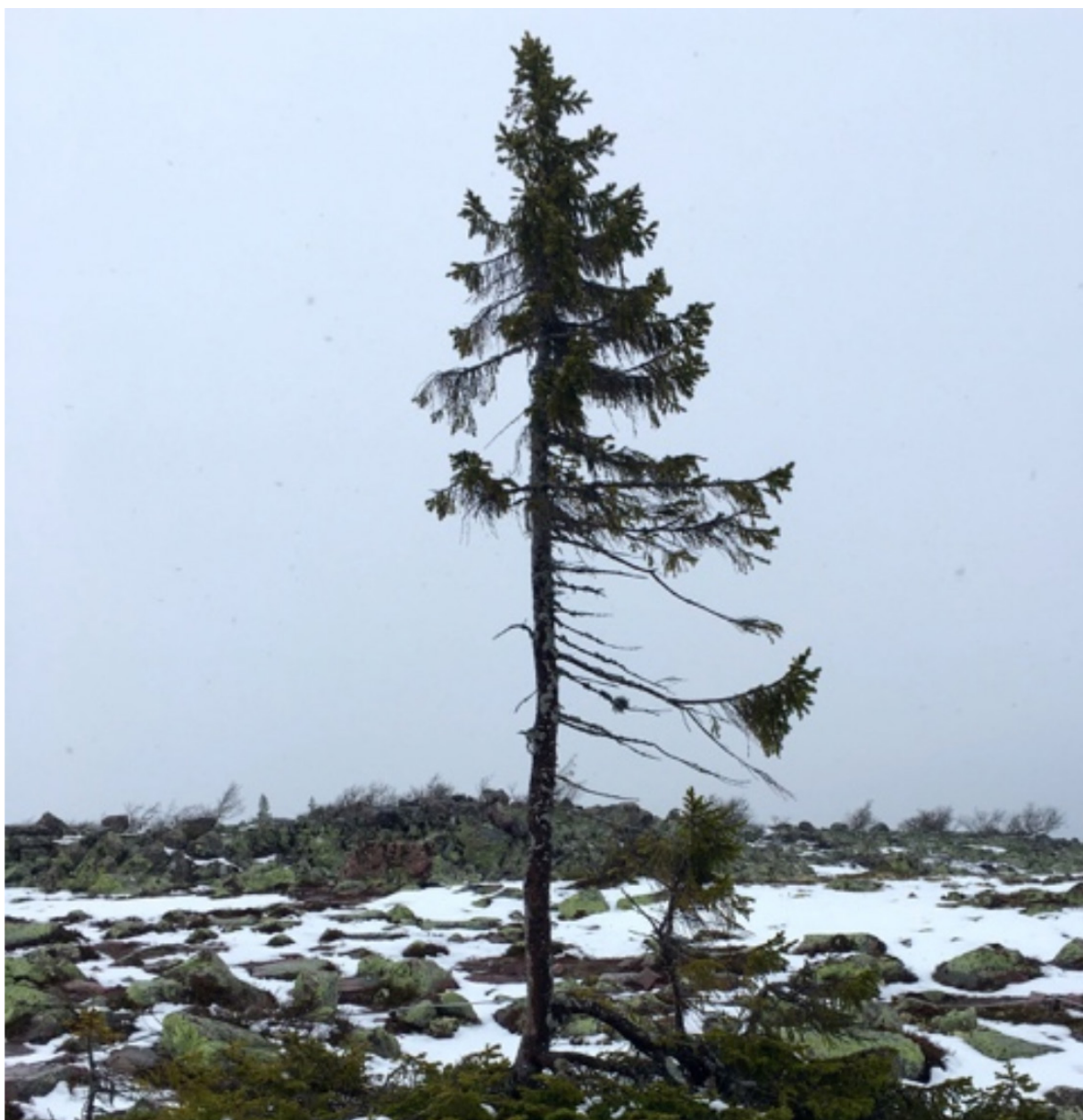

Figure 2: Old Tjikko is a 9,550-year-old Norway spruce clone, located on Fulufjället Mountain in the Dalarna province of Sweden. The visible part of the tree is less than 100 years old but is the last individual of a colony of trees with a very ancient root system (Photo credit: Laura Parducci).

P. abies trees are growing with plant material under their root systems dated up to $9.5 \mathrm{kyr}$ BP (Fig. 2). The early presence of tree species at high latitudes suggests either an early Holocene arrival or survival during the last glaciation. These hypotheses were tested using aDNA extracted from Norwegian lakes, and the genetic findings showed that spruce trees were present in Norway during the late glacial and the early Holocene (Parducci et al. 2012), leaving open, however, the question of their origin.

\section{Plant survival under changing climates}

These findings question traditional views on survival, resilience, and spread of cold-tolerant trees as a response to climate changes, and although restricted to specific regions and taxa, they are relevant to paleoecological studies worldwide. The ability of trees to survive at high latitudes has important implications for the response of forests to future climate changes and is particularly important for conservation and forest breeding programs interested in identifying genetic resources and provenances with traits related to survival under changing climates.

A first implication is the limited dispersal ability of certain forest tree species, which in turn implies that factors like geographical accessibility from glacial refugia and history are important constraints in species distribution (Svenning et al. 2008), and may act strongly as diversity predictors together with climate. It also implies that we cannot expect forests to simply track the forecasted 21st century climatic changes as suggested by several ecological niche modeling studies.

A second important implication is that postglacial migration rates based on pollen records require revision and should not be used alone to predict tree-range shifts in future modeling-based scenarios. Lower postglacial migration rates imply also that trees tolerate climatic changes better than previously assumed.

The scale of climate changes predicted for the next century is comparable to the scale of warming during the Holocene (several degrees Celsius depending on the region), but the rate of change is forecasted to be much faster (IPCC 2014). Recent modeling studies based on general circulation and vegetation models have suggested that rates of future climate changes require plant migration rates even faster than those observed in the fossil record, and hence may drastically reduce biodiversity by selecting for highly mobile and opportunistic plant species. However, the ability of certain species to tolerate changing climates and survive in northern refugia may mean that some catastrophic projections based on these models (Thuiller et al. 2005) might be overstated.

A final important implication for conservation is that northern populations may have preserved locally genotypes and genes best adapted to changing climatic conditions that are worth protecting. If trees only migrated from the south, the adaptation we see today to changes in temperature and photoperiod must have occurred over a relatively short time (the Holocene or the last $\sim 13,000$ years), and is based on "standing genetic variation" present in the southern macrorefugia, since this is a faster process than adaptation based on new mutations.

\section{New directions of plant aDNA research} Despite early challenges, plant aDNA research now offers the opportunity to detect hidden plant diversity and a complementary spectrum of fossil remains than those identified by traditional paleoecological analyses alone. In addition, new sequencing technologies have recently significantly changed the direction of plant aDNA research and we have moved from the analysis of a few chloroplast loci to the study of entire nuclear and organelle genomes. This has already provided important information for plant evolution, adaptation, conservation, crop breeding, and food security (Estrada et al. 2018). However, the aDNA field cannot work alone. Collaborative studies between researchers with different backgrounds (geologists, paleoecologists, archeologists, and molecular ecologists), as well as acknowledgment and discussion of the results from complementary disciplines, is not only a recommendation, but a requirement in this rapidly growing research field.

\section{AFFILIATION}

Department of Environmental Biology, La Sapienza University of Rome, Italy, and Department of Ecology and Genetics, Uppsala University, Sweden

\section{CONTACT}

Laura Parducci: Laura.Parducci@uniroma1.it

\section{REFERENCES}

Clark JS et al. (1998) BioScience 48: 13-24 Edwards ME et al. (2018) Holocene 28: 2006-2016 Estrada O et al. (2018) Nat Plants 4: 394-396 Feurdean A et al. (2013) PLoS ONE 8: e71797 Giguet-Covex C et al. (2019) Sci Rep 9: 14676 IPCC (2014) Pachauri RK, Meyer LA (Eds) Climate Change 2014: Synthesis Report. IPCC, 151 pp

Kullman L (2002) J Biogeogr 29: 1117-1124 Napier JD et al. (2019) Ecography 42: 1056-1067 Parducci L et al. (2012) Science 335: 1083-1086 Parducci L et al. (2017) New Phytol 214: $924-942$ Parducci L et al. (2019) Front Ecol Evol 7: 189 Pedersen MW et al. (2016) Nature 537: 45-49 Reid C (1899) The Origin of the British Flora. Dulau, $236 \mathrm{pp}$

Stewart JR et al. (2010) Proc Biol Sci B 277: 661-671 Svenning J-C et al. (2008) Ecography 31: 316-326 Taberlet P et al. (2018) Environmental DNA: For Biodiversity Research and Monitoring; Environmental DNA. Oxford University Press, $268 \mathrm{pp}$

Thuiller W et al. (2005) Proc Natl Acad Sci USA 102: 8245-8250

Tzedakis PC et al. (2013) Trends Ecol Evol 28: 696-704 Willerslev E et al. (2014) Nature 506: 47-51 